Pacific Journal of Mathematics

LOCALIZATIONS OF TORSION THEORIES

willy Brandal and Enol Barbet 


\title{
LOCALIZATIONS OF TORSION THEORIES
}

\author{
WILly BRANDAL AND EROL BARBUT
}

\begin{abstract}
If $\boldsymbol{R}$ is an $h$-local domain, then the hereditary torsion theories of $R$ are described in terms of the hereditary torsion theories of $R_{M}$ for all maximal ideals $M$ of $R$. By means of an example, it is shown that $h$-local is too strong a hypothesis for this localization property. As an application, all the hereditary torsion theories of $h$-local Prüfer domains are described. Some equivalent conditions for a domain to be $h$-local are generalized to conditions about hereditary torsion theories.
\end{abstract}

Introduction. $R$ will always denote an integral domain and all $R$-modules are unital modules. spec $R$ will denote the set of all prime ideals of $R$ and $\operatorname{mspec} R$ will denote the set of all maximal ideals of $R$. The main purpose of this paper is to describe the hereditary torsion theories of $R$ in terms of the hereditary torsion theories of the localizations $R_{M}$ for all $M \in \operatorname{mspec} R$. We will generally follow the terminology of the text by B. Stenström [9]. Because of the bijective correspondence between the hereditary torsion theories of $R$ and the Gabriel topologies of $R[9, \mathrm{Ch}$. 6, Theorem 5.1], it suffices to describe the Gabriel topologies of $R$. The results will be described mostly in terms of the Gabriel topologies of $R$.

We remind the reader of the definition. A Gabriel topology of $R$ is a non-empty family $\mathscr{F}$ of ideals of $R$ satisfying axioms T1-T4:

T1. If $I \in \mathscr{F}$ and $I \subset J$ for $J$ an ideal of $R$, then $J \in \mathscr{F}$.

T2. If $I, J \in \mathscr{F}$, then $I J \in \mathscr{F}$.

T3. If $I \in \mathscr{F}$ and $r \in R$, then $(I: r) \in \mathscr{F}$.

T4. If $I$ is an ideal of $R$ and $J \in \mathscr{F}$ with $(I: r) \in \mathscr{F}$ for all $r \in J$, then $I \in \mathscr{F}$.

The condition that $\mathscr{F}$ is non-empty is equivalent to requiring $R \in \mathcal{F}$. Note that condition $\mathrm{T} 2$ has been changed from the condition "if $I, J \in \mathscr{F}$, then $I \cap J \in \mathscr{F}$ " of the Stenström text [9] to the present equivalent form for commutative rings. It is easily seen that T3 and T4 imply T1 and T2, and since the rings considered in this paper are all commutative, T1 implies T3. Thus to show that $\mathscr{F}$ is a Gabriel topology of $R$ it suffices to verify $\mathrm{T} 3$ and $\mathrm{T} 4$, or to verify $\mathrm{T} 1$ and $\mathrm{T} 4$. It follows immediately from $\mathrm{T} 2$ that if $I \in \mathscr{F}$, then $I^{n} \in \mathscr{F}$ for all $n \geq 0$. Given a Gabriel topology $\mathscr{F}$ of $R$, the class of torsion $R$-modules of the corresponding hereditary torsion theory consists of all $R$-modules $T$ such that $\operatorname{Ann}_{R}(x) \in \mathscr{F}$ for all $x \in T$. 
1. Basic localization results. The following is given for the sake of easy reference.

LemMa 1.1. Let $S$ be a multiplicatively closed subset of $R, I$ an ideal of $R, J$ an ideal of $R_{S}$, and $r \in R$. Then:

(1) $(J \cap R)_{S}=J$.

(2) $I_{S} \cap R \supset I$.

(3) $\left(I_{S}: r\right) \supset(I: r)_{S}$.

(4) If $I_{S}=J$, then $I \subset J \cap R$.

(5) $(J \cap R: r)=(J: r) \cap R$.

(6) There exists a bijective order preserving correspondence between the set of all ideals of $R_{S}$ and a set of (some) ideals of $R$ given by $J \rightarrow J \cap R$ for $J$ an ideal of $R_{S}$.

Proof. Well known, omitted.

We introduce some notation. Let $S$ be a multiplicatively closed subset of $R$. If $\mathscr{F}$ is a Gabriel topology of $R$, let $\mathscr{F}_{S}$ denote $\left\{I_{S}: I \in \mathscr{F}\right\}$. If $\mathcal{G}$ is a Gabriel topology of $R_{S}$, let $\mathcal{G} \cap R$ denote $\{I: I$ is an ideal of $R$ and $\left.I_{S} \in \mathcal{G}\right\}$.

Proposition 1.2. Let $S$ be a multiplicatively closed subset of $R$ and $\mathscr{F} a$ Gabriel topology of $R$. Then $\mathscr{F}_{S}$ is a Gabriel topology of $R_{S}$.

Proof. Verify T1. Let $J \in \mathscr{F}_{S}$ and $J^{\prime}$ be an ideal of $R_{S}$ with $J \subset J^{\prime}$. Then $J=I_{S}$ for some $I \in \mathscr{F}$. By Lemma 1.1(2), $J^{\prime} \cap R \supset J \cap R=I_{S} \cap$ $R \supset I$, and so $J^{\prime} \cap R \in \mathscr{F}$ by T1 of $\mathscr{F}$. By Lemma 1.1(1), $J^{\prime}=\left(J^{\prime} \cap R\right)_{S}$, and so $J^{\prime} \in \mathscr{F}_{S}$, verifying T1.

Verify T4. Let $J$ be an ideal of $R_{S}$ with $L \in \mathscr{F}_{S}$ and $(J: y) \in \mathscr{F}_{S}$ for all $y \in L$. Then $L=I_{S}$ for some $I \in \mathscr{F}$. Since $I \subset I_{S}=L$, one has $(J: r) \in \mathscr{F}_{S}$ for all $r \in I$. Thus for each $r \in I$, there exists $I_{r} \in \mathscr{F}$ such that $\left(I_{r}\right)_{S}=(J: r)$. By Lemma 1.1(5) and (2), $(J \cap R: r)=(J: r) \cap R$ $=\left(I_{r}\right)_{S} \cap R \supset I_{r} \in \mathscr{F}$, and so $(J \cap R: r) \in \mathscr{F}$ by T1 of $\mathscr{F}$. Therefore $J \cap R \in \mathscr{F}$ by T4 of $\mathscr{F}$, and so $J=(J \cap R)_{S} \in \mathscr{F}_{S}$, verifying T4.

Proposition 1.3. Let $S$ be a multiplicatively closed subset of $R$ and $\mathcal{G} a$ Gabriel topology of $R_{S}$. Then $\mathcal{G} \cap R$ is a Gabriel topology of $R$.

Proof. Verify T3. Let $I \in \mathcal{G} \cap R$ and $r \in R$. Since $(I: r) \supset I,(I: r)_{S}$ $\supset I_{S} \in \mathcal{G}$, and so $(I: r) \in \mathcal{G} \cap R$ by $T 1$ of $\mathcal{G}$, verifying T3.

Verify T4. Let $I$ be an ideal of $R$ with $J \in \mathcal{G} \cap R$ and $(I: r) \in \mathcal{G} \cap R$ for all $r \in J$. By Lemma 1.1(3), $\left(I_{S}: r\right) \supset(I: r)_{S} \in \mathcal{G}$ and so $\left(I_{S}: r\right) \in \mathcal{G}$. Thus $\left(I_{S}: x\right) \in \mathcal{G}$ for all $x \in J_{S}$. By T4 of $\mathcal{G}, I_{S} \in \mathcal{G}$, and so $I \in \mathcal{G} \cap R$, verifying T4 for $\mathcal{G} \cap R$. 
Proposition 1.4. Let $S$ be a multiplicatively closed subset of $R$, and $\mathcal{G}$ a Gabriel topology of $R_{S}$. Then $(\mathcal{G} \cap R)_{S}=\mathcal{G}$.

Proof. Straightforward, using Lemma 1.1(1).

COROllary 1.5. Let $S$ be a multiplicatively closed subset of $R$. Then there exists a bijective order preserving correspondence between the set of all Gabriel topologies of $R_{S}$ and a set of (some) Gabriel topologies of $R$ given by $\mathcal{G} \rightarrow \mathcal{G} \cap R$ for $\mathcal{G}$ a Gabriel topology of $R$.

Proof. This is a translation of Proposition 1.4.

EXAMPLE 1.6. Let $R=Z$, the ring of integers, and let $S$ be the multiplicatively closed subset $S=Z-2 Z=\{x \in Z: 2$ does not divide $x\}$. Let $\mathscr{F}_{1}=\{Z\}$ and let $\mathscr{F}_{2}=\left\{3^{n} Z: n=0,1,2, \ldots\right\}$. Then $\mathscr{F}_{1}$ and $\mathscr{F}_{2}$ are Gabriel topologies of $Z$, and $\left(\mathscr{F}_{1}\right)_{S}=\left(\mathscr{F}_{2}\right)_{S}=\left\{Z_{S}\right\}$.

Lemma 1.1(6) gives the well-known bijective correspondence between the set of all ideals of $R_{S}$ and a set of (some) ideals of $R$, and in general this is not a bijective correspondence onto the set of all ideals of $R$. In a similar manner, Corollary 1.5 gives a bijective correspondence between the set of all Gabriel topologies of $R_{S}$ and a set of (some) Gabriel topologies of $R$, and by Example 1.6 this is in general not a bijective correspondence onto the set of all Gabriel topologies of $R$.

Lemma 1.7. Let $\varnothing \neq \Re \subset \operatorname{mspec} R$ and for $M \in \Re$ let $\mathcal{G}[M]$ be a Gabriel topology of $R_{M}$. Define $\mathscr{F}=\left\{I: I\right.$ is an ideal of $R$ and $I_{M} \in \mathcal{G}[M]$ for all $M \in \mathfrak{M}\}$. Then $\mathcal{F}$ is a Gabriel topology of $R$.

Proof. The intersection of a family of Gabriel topologies of $R$ is again a Gabriel topology of $R$. Hence this follows from Proposition 1.3.

2. $h$-local domains and the main results. $R$ is $h$-local if every non-zero prime ideal of $R$ is a subset of only one maximal ideal of $R$ and every non-zero element of $R$ is an element of only finitely many maximal ideals of $R$. This definition is due to E. Matlis [6] and discussion of $h$-local domains can be found in [6], [7], [8], [1], [2] and [4]. We briefly mention an important property of $h$-local domains. For an ideal $I$ of $R$, use the notation $\operatorname{mspec}(I)$ for $\{M \in \operatorname{mspec} R: I \subset M\}$ and for $r \in R$, use $\operatorname{mspec}(r)$ as an abbreviation of $\operatorname{mspec}(R r)$. In other words, $\operatorname{mspec}(I)=$ $V(I) \cap \operatorname{mspec} R$. Then a domain $R$ is $h$-local if and only if for each torsion $R$-module $T$ one has $T=\bigoplus_{M \in \operatorname{mspec} R} T(M)$ where $T(M)=\{x \in$ $\left.T: \operatorname{mspec}\left(\operatorname{Ann}_{R}(x)\right) \subset\{M\}\right\}$. Moreover, if $R$ is an $h$-local domain, $M \in$ mspec $R$, and $T$ a torsion $R$-module, then $T(M)$ and $T_{M}$ are isomorphic 
both as $R$-modules and as $R_{M}$-modules [4, proof of Theorem 2.6]. Thus $h$-local domains are exactly the domains whose torsion modules decompose into the direct sum of their " $M$-adic submodules" for all $M \in$ mspec $R$. This generalizes the fact that torsion Abelian groups decompose into the direct sum of their $p$-adic subgroups for all prime integers $p$. It would seem reasonable that the property of a domain being $h$-local is related to the torsion theories of the domain. Theorem 2.4 will illustrate this.

A non-zero Gabriel topology of $R$ is a Gabriel topology $\mathscr{F}$ of $R$ with the property that $\{0\} \notin \mathscr{F}$. In other words, a non-zero Gabriel topology of $R$ is one which is not the family of all ideals of $R$. Let $\mathcal{G}_{0}(R)$ denote the set of all non-zero Gabriel topologies of $R$. We will write the elements of $\Pi_{M \in \operatorname{mspec} R} \mathcal{G}_{0}\left(R_{M}\right)$ as tuples $\langle\mathcal{G}[M]\rangle$ where $\mathcal{G}[M] \in \mathcal{G}_{0}\left(R_{M}\right)$ for all $M \in$ mspec $R$. Define the function $\phi: \mathcal{G}_{0}(R) \rightarrow \prod_{M \in \operatorname{mspec} R} \mathcal{G}_{0}\left(R_{M}\right)$ by $\phi(\mathscr{F})=$ $\left\langle\mathscr{F}_{M}\right\rangle$ for $\mathscr{F} \in \mathcal{G}_{0}(R)$. By Proposition 1.2, $\mathscr{F}_{M}$ is an element of $\mathcal{G}_{0}\left(R_{M}\right)$.

LEMMA 2.1. Let $\mathscr{F} \in \mathcal{G}_{0}(R)$ and define $\mathscr{F}^{\prime}=\{I: I$ is an ideal of $R$ and $I_{M} \in \mathscr{F}_{M}$ for all $\left.M \in \operatorname{mspec} R\right\}$. Then $\mathscr{F}^{\prime} \in \mathcal{G}_{0}(R), \phi(\mathscr{F})=\phi\left(\mathscr{F}^{\prime}\right)$, and $\mathscr{F} \subset \mathscr{F}^{\prime}$. Furthermore, if $I \in \mathscr{F}^{\prime}$ and $I$ is a subset of only finitely many maximal ideals of $R$, then $I \in \mathcal{F}$.

Proof. By Lemma 1.7, $\mathscr{F}^{\prime} \in \mathcal{G}_{0}(R)$, and clearly $\mathscr{F} \subset \mathscr{F}^{\prime}$. Let $M \in$ mspec $R$. To verify that $\phi(\mathscr{F})=\phi\left(\mathscr{F}^{\prime}\right)$, it will be shown that $\mathscr{F}_{M}=\mathscr{F}_{M}^{\prime}$. Let $J \in \mathscr{F}_{M}$, hence $J=I_{M}$ for some $I \in \mathscr{F}$. Then $I \in \mathscr{F}^{\prime}$, and $J=I_{M} \in$ $\mathscr{F}_{M}^{\prime}$. On the other hand suppose $J \in \mathscr{F}_{M}^{\prime}$, hence $J=I_{M}$ for some $I \in \mathscr{F}^{\prime}$. Therefore $J=I_{M} \in \mathscr{F}_{M}$ by definition of $\mathscr{F}^{\prime}$. This verifies that $\phi(\mathscr{F})=$ $\phi\left(\mathscr{F}^{\prime}\right)$.

Suppose $I \in \mathscr{F}^{\prime}$ and $I$ is a subset of only finitely many maximal ideals of $R$, say $M_{1}, \ldots, M_{n}$. Then $I_{M_{t}} \in \mathscr{F}_{M_{i}}$, and so $I_{M_{i}}=\left(J_{i}\right)_{M_{t}}$ for some $J_{l} \in \mathscr{F}_{\text {. }}$. Since $J_{i} \subset\left(J_{l}\right)_{M_{i}} \cap R=I_{M_{t}} \cap R$, we have $I_{M_{t}} \cap R \in \mathscr{F}$. Because $I_{M}=R_{M}$ for all $M \in \operatorname{mspec} R-\left\{M_{1}, \ldots, M_{n}\right\}$, and $I=\bigcap_{M \in \operatorname{mspec} R} I_{M}$, we have $I=\cap_{l=1}^{n}\left(I_{M_{i}} \cap R\right)$. Hence, by $\mathrm{T} 1$ and $\mathrm{T} 2, I \in \mathscr{F}$.

COROLlARY 2.2. If every non-zero ideal of $R$ is a subset of only finitely many maximal ideals of $R$, then $\phi$ is injective.

Proof. Let $\mathscr{F}_{1}, \mathscr{F}_{2} \in \mathcal{G}_{0}(R)$ and suppose $\phi\left(\mathscr{F}_{1}\right)=\phi\left(\mathscr{F}_{2}\right)$. By Lemma 2.1, $\mathscr{F}_{1}=\mathscr{F}_{1}^{\prime}$ and $\mathscr{F}_{2}=\mathscr{F}_{2}^{\prime}$. Also $\left(\mathscr{F}_{1}\right)_{M}=\left(\mathscr{F}_{2}\right)_{M}$ for all $M \in \operatorname{mspec} R$, and so $\mathscr{F}_{1}^{\prime}=\mathscr{F}_{2}^{\prime}$. Thus $\mathscr{F}_{1}=\mathscr{F}_{2}$, and $\phi$ is injective.

Lemma 2.3. Every non-zero prime ideal of $R$ is a subset of only one maximal ideal of $R$ if and only if $\phi$ is surjective. 
Proof. Assume that every non-zero prime ideal of $R$ is a subset of only one maximal ideal of $R$. Let $\langle\mathcal{G}[M]\rangle \in \Pi_{M \in \operatorname{mspec} R} \mathcal{G}_{0}\left(R_{M}\right)$. Define $\mathscr{F}=$ $\left\{I: I\right.$ is an ideal of $R$ and $I_{M} \in \mathcal{G}[M]$ for all $\left.M \in \operatorname{mspec} R\right\}$. By Lemma $1.7, \mathscr{F} \in \mathcal{G}_{0}(R)$. Let $M \in \operatorname{mspec} R$. We must verify that $\mathscr{F}_{M}=\mathcal{G}[M]$. Clearly $\mathscr{F}_{M} \subset \mathcal{G}[M]$. On the other hand, let $J \in \mathcal{G}[M]$ and let $I=J \cap R$. Then $I_{M}=J \in \mathcal{G}[M]$ by Lemma 1.1(1). Let $P \in \operatorname{mspec} R-\{M\}$. Since $P \cap M$ contains no non-zero prime ideal of $R$, we have $J_{P}=Q$, the quotient field of $R$. Thus $I_{P}=J_{P} \cap R_{P}=Q \cap R_{P}=R_{P} \in \mathcal{G}[P]$. Therefore $I \in \mathscr{F}$ by definition of $\mathscr{F}$, and so $J=I_{M} \in \mathscr{F}_{M}$.

Conversely, suppose that $R$ is a domain with a non-zero prime ideal $P$ such that $P \subset M_{1} \cap M_{2}$ with $M_{1}, M_{2} \in \operatorname{mspec} R$ and $M_{1} \neq M_{2}$. Let $\mathscr{F}$ be the Gabriel topology of $R$ given by $\mathscr{F}=\{I: I$ is an ideal of $R$ and $P \not \supset I\}$. For $M \in \operatorname{mspec} R-\left\{M_{2}\right\}$, let $\mathcal{G}[M]=\mathscr{F}_{M}$. Let $\mathcal{G}\left[M_{2}\right]=\{J: J$ is a nonzero ideal of $\left.R_{M_{2}}\right\}$. Then $\langle\mathcal{G}[M]\rangle \in \Pi_{M \in \operatorname{mspec} R} \mathcal{G}_{0}\left(R_{M}\right)$ and we shall show that $\langle\mathcal{G}[M]\rangle$ is not in the image of $\phi$.

Suppose $\mathscr{F}_{1} \in \mathcal{G}_{0}(R)$ and $\phi\left(\mathscr{F}_{1}\right)=\langle\mathcal{G}[M]\rangle$. Then $\left(\mathscr{F}_{1}\right)_{M}=\mathscr{F}_{M}$ for all $M \in \operatorname{mspec} R-\left\{M_{2}\right\}$ and $\left(\mathscr{F}_{1}\right)_{M_{2}}=\mathcal{G}\left[M_{2}\right]$. Now $P_{M_{2}} \in \mathcal{G}\left[M_{2}\right]$ and hence $P=P_{M_{2}} \cap R \in \mathscr{F}_{1}$. Therefore $P_{M_{1}} \in\left(\mathscr{F}_{1}\right)_{M_{1}}=\mathcal{G}\left[M_{1}\right]$ and $P=P_{M_{1}} \cap R$ $\in \mathscr{F}$ and so $P \not \supset P$. This contradiction shows that $\phi$ is not surjective.

THEOREM 2.4. If $R$ is an h-local domain, then $\phi$ is a bijection.

Proof. This is a summary of Corollary 2.2 and Lemma 2.3 .

THEOREM 2.5. Let $R$ be an h-local domain. Then $\mathcal{T}$ is the torsion class of a hereditary torsion theory of $R$ if and only if either

1. $\mathcal{F}$ is the class of all $R$-modules or

2. for all $M \in \operatorname{mspec} R$ there exists a torsion class $\mathcal{T}[M]$ of a hereditary torsion theory of $R_{M}$ that is not the class of all $R_{M}$-modules, and $T \in \mathcal{T}$ if and only if $T_{M} \in \mathcal{T}[M]$ for all $M \in \operatorname{mspec} R$.

Proof. This is a translation of Theorem 2.4 to torsion theories.

The above results say that for $h$-local domains, a hereditary torsion theory of $R$ is determined by its localizations, i.e., by the corresponding hereditary torsion theory for $R_{M}$ for all $M \in \operatorname{mspec} R$. One might suspect that this localization property characterizes the $h$-local domains. This is not quite the case as the following example indicates.

EXAMPLE 2.6. There exists a domain $R$ with the property that $R$ is not $h$-local and $\phi$ is a set bijection. 
Proof. We will use the Krull-Kaplansky-Jaffard-Ohm method of constructing Bezout domains as described in [3]. The example to be given is due to W. Heinzer and $\mathrm{J}$. Ohm [5] (also in [3]). Let $Z$ be the totally ordered group of integers, let $N$ be the set of positive integers, let $Z^{N}$ have the product ordering, and view elements of $Z^{N}$ as sequences $\left\langle z_{1}, z_{2}, \ldots\right\rangle$ or simply $\left\langle z_{n}\right\rangle$ where $z_{n} \in Z$ for all $n \in N$. Let $G=\left\{\left\langle z_{n}\right\rangle \in Z^{N}\right.$ : there exists $k \in Z$ such that $z_{n}=k$ for all but finitely many $\left.n \in N\right\}$. With the induced ordering of $Z^{N}, G$ becomes a lattice ordered group. By [3, Theorem 1.9] there exists a Bezout domain $R$ whose group of divisibility is order isomorphic to $G$. Let $Q$ be the quotient field of $R$ and use an asterisk* to denote non-zero elements. Then there exists a surjective function $\pi: Q^{*} \rightarrow G$ so that $R=\{0\} \cup\left\{x \in Q^{*}: \pi(x) \geq 0\right\}$. For $i \in N$, let $M_{l}=\{0\} \cup\left\{r \in R^{*}:\right.$ if $\pi(r)=\left\langle z_{n}\right\rangle$, then $\left.z_{l}>0\right\}$, and let $M_{\infty}=\{0\}$ $\cup\left\{r \in R^{*}:\right.$ if $\pi(r)=\left\langle z_{n}\right\rangle$, then $z_{n}>0$ for all but finitely many $\left.n \in N\right\}$. Then mspec $R=\left\{M_{n}\right\}_{n \in N \cup\{\infty\}}$ and $R_{M}$ is a discrete valuation ring (DVR) for all $M \in \operatorname{mspec} R$. It will be shown that this $R$ has the required properties.

Every non-zero element of $M_{\infty}$ is an element of infinitely many maximal ideals of $R$, so $R$ is not $h$-local.

Since $\operatorname{spec} R=\operatorname{mspec} R \cup\{\{0\}\}$, every non-zero prime ideal of $R$ is a subset of only one maximal ideal of $R$. By Lemma $2.3, \phi$ is a surjective function. To verify that $\phi$ is injective, consider $\langle\mathcal{G}[M]\rangle \in$ $\Pi_{M \in \operatorname{mspec} R} \mathcal{G}_{0}\left(R_{M}\right)$. Since $R_{M}$ is a DVR for all $M \in \operatorname{mspec} R$, there are only two possibilities for $\mathcal{G}[M]$, namely $\left\{R_{M}\right\}$ or $\left\{M_{M}^{k}\right\}_{k=0}^{\infty}$. Define $\Re=\left\{M \in \operatorname{mspec} R: \mathcal{G}[M]=\left\{M_{M}^{k}\right\}_{k=0}^{\infty}\right\}$. Let $\mathscr{F} \in \mathcal{G}_{0}(R)$ be such that $\phi(\mathscr{F})=\langle\mathcal{G}[M]\rangle$.

We claim that $\Re \subset \mathscr{F}$. For if $M \in \mathfrak{N}$, then $M_{M} \in \mathcal{G}[M]$ so there exists $I \in \mathscr{F}$ with $I_{M}=M_{M}$. But then $I \subset M$ and so by T1, $M \in \mathscr{F}$. This verifies the claim $\mathscr{N} \subset \mathcal{F}$. Three cases will be considered.

Case 1. Suppose $M_{\infty} \notin \Re$. We claim that $\mathscr{F}$ is the set of all finite products of the elements of $\Re$ (including $R$, the empty product). By T2, F contains all finite products of elements of $\Re$. On the other hand, let $I \in \mathscr{F}$. Since $M_{\infty} \notin \Re$, we have $I_{M_{\infty}}=R_{M_{\infty}}$. Thus there exists $x \in I-$ $M_{\infty}$. If $\pi(x)=\left\langle z_{n}\right\rangle$, then $\operatorname{mspec}(x)=\left\{M_{i} \in \operatorname{mspec} R: z_{l}>0\right\}$, and so $\operatorname{mspec}(x)$ is a finite set. Let $\operatorname{mspec}(x)=\left\{M_{i_{1}}, \ldots, M_{\iota_{n}}\right\}$. For $j=1,2, \ldots, n$ there is a positive integer $z_{l_{1}}$ so that $R_{M_{i}} x=\left(M_{l_{1}}\right)_{M_{i}}^{z_{l_{1}}}$. Then $I \supset R x=M_{l_{1}}^{z_{l_{1}}}$ $\cdots M_{l_{n}}^{z_{l_{n}}}$, and so it follows that $I$ is of the required form. This verifies that $\mathscr{F}$ is the set of all finite products of elements of $\mathfrak{K}$.

Case 2. Suppose $M_{\infty} \in \Re$ and $\Re$ is finite. We claim that $\mathscr{F}$ is the set of all finite products of elements of $\Re$. As in Case 1, $\mathscr{F}$ contains all finite 
products of elements of $\mathfrak{T}$. Suppose $\mathfrak{T}=\left\{M_{\infty}, M_{i_{1}}, \ldots, M_{i_{n}}\right\}$. Let $I \in \mathscr{F}$. Then there exist non-negative integers $k, k_{1}, \ldots, k_{n}$ such that $I_{M_{\infty}}=$ $\left(M_{\infty}\right)_{M_{\infty}}^{k}$ and $I_{M_{j}}=\left(M_{i_{j}}\right)_{M_{i^{\prime}}}^{k_{j}}$ for $j=1,2, \ldots, n$. Therefore $I=M_{\infty}^{k} M_{i_{1}}^{k_{1}}$ $\cdots M_{i_{n}}^{k_{n}}$, which verifies the claim that $\mathscr{F}$ is the set of all finite products of elements of $\mathfrak{N}$.

Case 3. Suppose $M_{\infty} \in \mathfrak{R}$ and $\mathscr{T}$ is infinite. Define $\mathscr{F}_{0}=\{I: I$ is an ideal of $R$ and $I_{M} \in \mathcal{G}[M]$ for all $\left.M \in \operatorname{mspec} R\right\}$. We claim that $\mathscr{F}=\mathscr{F}_{0}$. Let $I_{0}=\cap \mathscr{R}$. We first show that $I_{0} \in \mathscr{F}$. Let $x \in M_{\infty}$ and consider $\left(I_{0}: x\right)$. Let $\left\{M_{i_{1}}, \ldots, M_{\imath_{m}}\right\}=\{M \in \mathfrak{N}: x \notin M\}=\mathscr{N}-\operatorname{mspec}(x)$. Then $\left(I_{0}: x\right) \supset M_{i_{1}} M_{t_{2}} \cdots M_{i_{n}} \in \mathscr{F}$, and so $\left(I_{0}: x\right) \in \mathscr{F}$ for all $x \in M_{\infty}$ $\in \mathscr{F}$. By T4, it follows that $I_{0} \in \mathscr{F}$. To verify the claim $\mathscr{F}=\mathscr{F}_{0}$, we first consider $I \in \mathscr{F}_{0}$. There exists a non-negative integer $k$ so that $I_{M_{\infty}}=$ $\left(M_{\infty}\right)_{M_{\infty}}^{k}$. There exists $x \in I$ such that $R_{M_{\infty}} x=\left(M_{\infty}\right)_{M_{\infty}}^{k}$. Suppose $\pi(x)=$ $\left\langle z_{n}\right\rangle .{ }^{\infty}$ Let $\left\{M_{i_{1}}, \ldots, M_{i_{n}}\right\}=\left\{M_{i} \in \Re_{R}: z_{\imath} \stackrel{>}{>} k\right\}=\{M \in$ IT: $\left.R_{M} x \subsetneq M_{M}^{k}\right\}$. Then $I \supset I_{0}^{k} M_{i_{1}}^{z_{t_{1}}-k} M_{i_{2}}^{z_{l_{2}}-k} \cdots M_{i_{n}}^{z_{i_{n}}-k} \in \mathscr{F}$, and so $I \in$ $\mathscr{F}$. Thus $\mathscr{F}_{0} \subset \mathscr{F}$. Since $\phi(\mathscr{F})=\langle\mathcal{G}[M]\rangle$, one has $\mathscr{F} \stackrel{\mathscr{F}^{n}}{\subset}$. This verifies the claim that $\mathscr{F}=\mathscr{F}_{0}$.

In all three cases, there is a description of $\mathscr{F}$ in terms of the given $\langle\mathcal{G}[M]\rangle$, and so only one such $\mathscr{F}$ is possible. Thus $\phi$ is injective.

The discussion in this section does not fully answer the question of when the function $\phi$ is a bijection, i.e., when Gabriel topologies are described locally. When $R$ is an $h$-local domain, then $\phi$ is a bijection by Theorem 2.4, but $h$-local is too strong a hypothesis by Example 2.6. It is possible that $\phi$ is a bijection if and only if every non-zero prime ideal of $R$ is a subset of only one maximal ideal of $R$. It is also possible that one needs an additional condition that is weaker than requiring that every non-zero element of $R$ is an element of only finitely many maximal ideals of $R$.

3. Valuation domains and other examples. We wish to describe all the Gabriel topologies of valuation domains. Following the B. Stenström text [9], the following notation will be used. For $P \in \operatorname{spec} R$, let $\mathcal{F}(P)$ be $\{I: I$ is an ideal of $R$ and $I \not \subset P\}$, and for $\mathscr{P} \subset \operatorname{spec} R$, let $\mathscr{F}(\mathscr{P})=\bigcap_{P \in \mathcal{P}} \mathscr{F}(P)$. Then $\mathscr{F}(P)$ and $\mathscr{F}(\mathcal{P})$ are Gabriel topologies of $R$. Moreover, in some special cases such as if $R$ is a Noetherian ring, then every Gabriel topology of $R$ is of the form $\mathscr{F}(\mathcal{P})$ for some $\mathcal{P} \subset \operatorname{spec} R$ [9, Ch. 6. Corollary 6.15]. As shall be seen, the situation is not this simple for valuation domains. We remark that if $P$ is an idempotent ideal of $R$, i.e., $P^{2}=P$, then $\{I: I$ is an ideal of $R$ and $P \subset I\}$ is a Gabriel topology of $R[9$, Ch. 6, Proposition $6.11]$. 
EXAMPLE 3.1. There exists a valuation domain $R$ with a Gabriel topology which is not of the form $\mathscr{F}(\mathscr{P})$ for some $\mathscr{P} \subset \operatorname{spec} R$.

Proof. Let $R$ be a valuation domain whose group of divisibility (i.e., value group) is order isomorphic to the additive group of rationals with the standard ordering (for example [10, page 101, Example 2-use rational instead of real exponents] which is the same as [4, Example 12.2]). If $M$ is the maximal ideal of $R$, then $M^{2}=M$ and $\operatorname{spec} R=\{M,\{0\}\}$. Let $\mathscr{F}=\{R, M\}=\{I: I$ is an ideal of $R$ and $I \supset M\}$. Then $\mathscr{F}$ is a Gabriel topology of $R$ and $\mathscr{F} \neq \mathscr{F}(\mathscr{P})$ for any $\mathscr{P} \subset \operatorname{spec} R$.

We present an alternate description of the Gabriel topologies $\mathscr{F}(\mathscr{P})$ for $\mathscr{P} \subset \operatorname{spec} R$ where $R$ is a valuation domain, and then describe all the Gabriel topologies of a valuation domain.

Proposition 3.2. Let $R$ be a valuation domain and $\mathscr{P} \subset \operatorname{spec} R$. Define $P=\cup \mathcal{P}$. Then $P \in \operatorname{spec} R$ and there are two possibilities for $\mathscr{F}(\mathscr{P})$ :

1. If $P \in \mathscr{P}$, then $\mathscr{F}(\mathscr{P})=\mathscr{F}(P)=\{I: I$ is an ideal of $R$ and $P \subsetneq I\}$.

2. If $P \notin \mathscr{P}$, then $\mathscr{F}(\mathscr{P})=\mathscr{F}(P) \cup\{P\}=\{I: I$ is an ideal of $R$ and $P \subset I\}$.

Proof. 1. This follows from the fact that if $P_{1}, P_{2} \in \operatorname{spec} R$ and $P_{1} \supset P_{2}$, then $\mathscr{F}\left(P_{1}\right) \subset \mathscr{F}\left(P_{2}\right)$.

2. Since $P \notin \mathscr{P}$, one has $P \in \bigcap_{J \in \mathscr{P}} \mathscr{F}(J)=\mathscr{F}(\mathscr{P})$ and so $\mathscr{F}(\mathscr{P}) \supset$ $\mathscr{F}(P) \cup\{P\}$. The other inclusion is straightforward since $R$ is a valuation domain.

THEOREM 3.3. Let $R$ be a valuation domain. Then $\mathscr{F}$ is a Gabriel topology of $R$ if and only if either

1. there exists $P \in \operatorname{spec} R$ and $\mathscr{F}=\mathscr{F}(P)$, or

2. there exists $P \in \operatorname{spec} R$ with $P^{2}=P$ and $\mathscr{F}=\mathscr{F}(P) \cup\{P\}$.

Proof. It has already been noted that the two types of $\mathscr{F}$ described are Gabriel topologies. Conversely, suppose $\mathscr{F}$ is a Gabriel topology of $R$. Define $P=\cup\left\{P^{\prime} \in \operatorname{spec} R: P^{\prime} \subset \cap \mathscr{F}\right\}$. Then $P \in \operatorname{spec} R$ and $I \supset P$ for all $I \in \mathscr{F}$. Suppose $I_{0}$ is an ideal of $R$ and $I_{0} \supsetneqq P$. We claim that $I_{0} \in \mathscr{F}$.

Define $P_{1}=\bigcap\left\{P^{\prime} \in \operatorname{spec} R: P^{\prime} \supset I_{0}\right\}$. Then $P_{1} \in \operatorname{spec} R$ and $P_{1} \supset$ $I_{0} \supsetneqq P$. Therefore $P_{1} \not \subset \cap \mathscr{F}$ and so there exists $J \in \mathscr{F}$ with $P_{1} \not \subset J$. Hence $J \subsetneq P_{1}$, and choose $x \in P_{1}-J$. Then $J \subsetneq R x$ and so $R x \in \mathscr{F}$. Let $P_{2}=\cap_{n=1}^{\infty} R x^{n}$. Then $P_{2} \in \operatorname{spec} R$ and $P_{1} \supsetneqq P_{2}$. It follows that $P_{2} \subsetneq I_{0}$. There exists an integer $n>0$ with $R x^{n} \subsetneq I_{0}$. Since $R x \in \mathscr{F}$, we have $R x^{n} \in \mathscr{F}$ by T2. Thus $I_{0} \in \mathcal{F}$, as claimed. 
Therefore $\mathscr{F}$ must be $\mathscr{F}(P)$ or $\mathscr{F}(P) \cup\{P\}$. In the latter case, $P \in \mathscr{F}$ implies $P^{2} \in \mathscr{F}$ by $\mathrm{T} 2$, and so $P^{2}=P$.

Consider the Example 3.1 where $R$ is a valuation domain with maximal ideal $M$ and $M^{2}=M$ and $\operatorname{spec} R=\{M,\{0\}\}$. Then by Theorem 3.3, there are four Gabriel topologies of $R$, namely $\mathscr{F}_{1}=\{R\}, \mathscr{F}_{2}=$ $\{R, M\}, \mathscr{F}_{3}$ the set of all non-zero ideals of $R$, and $\mathscr{F}_{4}$ the set of all ideals of $R$. One can readily describe the corresponding hereditary torsion theories. For $\mathscr{F}_{1}$, the only torsion $R$-module is the zero module. For $\mathscr{F}_{2}$, the torsion $R$-modules are the semi-simple $R$-modules. For $\mathscr{F}_{3}$, the torsion $R$-modules are the "classical torsion modules," i.e., the $R$-modules $T$ where $\operatorname{Ann}_{R}(x) \neq\{0\}$ for all $x \in T$. For $\mathscr{F}_{4}$, every $R$-module is torsion.

As a comparison, consider the example $R$ which is a DVR with maximal ideal $M$. Then there are three Gabriel topologies of $R$, namely $\mathscr{F}_{1}, \mathscr{F}_{3}$, and $\mathscr{F}_{4}$ as described in the last paragraph, with the same corresponding torsion theories. Relating these to $\mathscr{F}(\mathscr{P})$ for $\mathscr{P} \subset \operatorname{spec} R$, one has $\mathscr{F}_{1}=\mathscr{F}(\{M\})=\mathscr{F}(\operatorname{spec} R), \mathscr{F}_{3}=\mathscr{F}(\{\{0\}\})$ and $\mathscr{F}_{4}=\mathscr{F}(\varnothing)$.

We briefly comment on another difference of these two Krull dimension one valuation domains in the last two paragraphs. By [9, Ch. 6, Proposition 2.1] if $\mathcal{T}$ is the torsion class of some torsion theory, then $\mathcal{T}$ is closed under extensions. Thus, for the $R$ of Example 3.1, one has the fact that if $0 \rightarrow S_{1} \rightarrow S_{2} \rightarrow S_{3} \rightarrow 0$ is a short exact sequence of $R$-modules and $S_{1}$ and $S_{3}$ are semi-simple, then $S_{2}$ is semi-simple, and so the sequence splits.In other words, $\operatorname{Ext}_{R}^{1}\left(S_{3}, S_{1}\right) \cong\{0\}$ for all semi-simple $R$-modules $S_{3}$ and $S_{1}$. On the other hand, if $R$ is a DVR with maximal ideal $M$, then if $0 \rightarrow R / M \rightarrow A \rightarrow R / M \rightarrow 0$ is a short exact sequence of $R$-modules, this does not imply that the sequence splits-one can have it not split with $A=R / M^{2}$. In other words, $\operatorname{Ext}_{R}^{1}(R / M, R / M) \vDash\{0\}$.

Recall that $R$ is a Prüfer domain if and only if $R_{M}$ is a valuation domain for all $M \in \operatorname{mspec} R$.

Theorem 3.4. Let $R$ be an h-local Prüfer domain. Then $\mathcal{T}$ is the torsion class of a hereditary torsion theory of $R$ if and only if either

1. $\mathcal{T}$ is the class of all $R$-modules or

2. $\mathcal{T}$ is the class of all $R$-modules $T$ satisfying $T_{M} \in \mathcal{T}[M]$ for all $M \in \operatorname{mspec} R$, where for each $M \in \operatorname{mspec} R$ one has $\mathcal{T}[M]$ given by either

(a) there exists $P \in \operatorname{spec} R_{M}$ and $\mathscr{T}[M]$ is the class of all $R_{M}$-modules $U$ such that $\operatorname{Ann}_{R_{M}}(x) \supsetneqq P$ for all $x \in U$ or

(b) there exists $P \in \operatorname{spec} R_{M}$ with $P^{2}=P \neq\{0\}$ and $\mathcal{T}[M]$ is the class of all $R_{M}$-modules $U$ such that $\mathrm{Ann}_{R_{M}}(x) \supset P$ for all $x \in U$.

Proof. This is a combination of Theorem 2.5 and Theorem 3.3. 
4. More on $h$-local domains. For $\mathscr{F}$ a Gabriel topology of $R$, the corresponding class of torsion $R$-modules will be called $\mathscr{F}$-torsion $R$-modules. Thus $T$ is an $\mathscr{F}$-torsion $R$-module if and only if $\operatorname{Ann}_{R}(x) \in \mathscr{F}$ for all $x \in T$.

THEOREM 4.1. Let $\mathscr{P} \subset \operatorname{mspec} R$ and consider $\mathscr{F}=\mathscr{F}(\{\{0\}\} \cup$ (mspec $R-\mathcal{P})$ ). The following statements are equivalent:

1. For all $x \in R-\{0\}$ with $\operatorname{mspec}(x) \subset \mathscr{P}$ one has $\operatorname{mspec}(x)$ is finite and for all $P \in \operatorname{spec} R-\{\{0\}\}$ with $\operatorname{mspec}(P) \subset \mathcal{P}$ one has $P$ is a subset of only one maximal ideal of $R$.

2. $T=\bigoplus_{M \in \operatorname{mspec} R} T(M)$ for every $\mathscr{F}$-torsion $R$-module $T$.

3. $T=\bigoplus_{M \in \mathcal{P}} T(M)$ for every $\mathcal{F}$-torsion $R$-module $T$.

4. $T=\bigoplus_{M \in \operatorname{mspec} R} T(M)$ for every cyclic $\mathscr{F}$-torsion $R$-module $T$.

5. $T=\bigoplus_{M \in \mathcal{P}} T(M)$ for every cyclic $\mathcal{F}$-torsion $R$-module $T$.

Proof. Let $T$ be an $\mathscr{F}$-torsion $R$-module. We claim that $T(M)=\{0\}$ for all $M \in \operatorname{mspec} R-\mathscr{P}$. One has $\operatorname{Ann}_{R}(x) \in \mathscr{F}$ for all $x \in T$. Then $V\left(\operatorname{Ann}_{R}(x)\right) \cap(\{\{0\}\} \cup(\operatorname{mspec} R-\mathscr{P}))=\varnothing$, and so $\operatorname{Ann}_{R}(x) \neq\{0\}$ and $\operatorname{Ann}_{R}(x) \not \subset M$ for all $M \in \operatorname{mspec} R-\mathscr{P}$. Hence $T(M)=\{0\}$ for all $M \in \operatorname{mspec} R-\mathcal{P}$, as claimed.

$2 \leftrightarrow 3$. Follows from the above paragraph.

$4 \leftrightarrow 5$. Follows from the above paragraph.

$1 \rightarrow 3$. Let $T$ be an $\mathscr{F}$-torsion $R$-module and let $x \in T$. Then $\operatorname{mspec}\left(\operatorname{Ann}_{R}(x)\right) \subset \mathcal{P}$ by the first paragraph. By Condition 1, $\operatorname{mspec}\left(\operatorname{Ann}_{R}(x)\right)$ is finite. By [4, Lemma 2.4] $R x \cong R / \operatorname{Ann}_{R}(x)$ is a finite direct sum of indecomposable submodules, i.e., $R x=\bigoplus_{l=1}^{n} R x_{l}$. Then

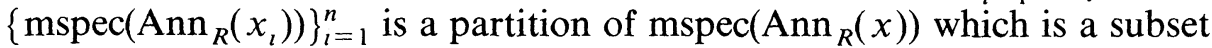
of $\mathscr{P}$. By [4, Proposition 2.5] and the second half of Condition 1, it must be the case that $\operatorname{mspec}\left(\operatorname{Ann}_{R}\left(x_{i}\right)\right)$ has at most one element for each $i=1,2, \ldots, n$. Thus $R x_{i} \subset T(M)$ for some $M \in \mathscr{P}$. This implies that $x \in \Sigma_{M \in \mathscr{P}} T(M)$, i.e., $T=\Sigma_{M \in \mathscr{P}} T(M)$. It is straightforward to show that the sum is direct.

$3 \rightarrow 5$. Trivial.

$5 \rightarrow 1$. Let $x \in R-\{0\}$ with $\operatorname{mspec}(x) \subset \mathscr{P}$. Consider $T=R / R x$. Then $\operatorname{Ann}_{R}(T) \neq\{0\}$ and $\operatorname{Ann}_{R}(T) \not \subset M$ for all $M \in \operatorname{mspec} R-\mathscr{P}$, and so $T$ is a cyclic $\mathcal{F}$-torsion $R$-module. By Condition $5, T=\bigoplus_{M \in \mathcal{P}} T(M)$. Since $T$ is a cyclic $R$-module, $T$ must be a finite direct sum, and so $\operatorname{mspec}(x)$ is finite. Let $P \in \operatorname{spec} R-\{\{0\}\}$ with $\operatorname{mspec}(P) \subset \mathscr{P}$. Consider $U=R / P$. As above, $U$ is a cyclic $\mathscr{F}$-torsion $R$-module. By Condition 5, $U=\bigoplus_{M \in \mathcal{P}} U(M)$. But since $P \in \operatorname{spec} R$, it must be the case that $U$ is indecomposable. Thus $P$ is a subset of only one maximal ideal of $R$. 
COROLlaRy 4.2. [E. Matlis]. The following statements are equivalent.

1. $R$ is an h-local domain.

2. $T=\bigoplus_{M \in \operatorname{mspec} R} T(M)$ for every torsion $R$-module.

3. $T=\bigoplus_{M \in \operatorname{mspec} R} T(M)$ for every cyclic torsion $R$-module.

Proof. This is the special case of Theorem 4.1 with $\mathscr{P}=\operatorname{mspec} R$.

Since Corollary 4.2 is a special case of Theorem 4.1 , one can view the five conditions of this theorem as generalizations of the definition of $h$-local domains. Each instance of the generalization comes from a Gabriel topology $\mathscr{F}$ of the type given in the hypothesis of Theorem 4.1.

The authors thank the referee for several suggestions that improved the organization of this paper.

\section{REFERENCES}

1. W. Brandal, Almost maximal integral domains and finitely generated modules, Trans. Amer. Math. Soc., 183 (1973), 203-222.

2. __ On h-local integral domains, Trans. Amer. Math. Soc., 206 (1975), 201-212.

3. __ Constructing Bezout domains, Rocky Mountain Math. J., 6 (1976), 383-399.

$4 . \quad$ Commutative rings whose finitely generated modules decompose, Lecture Notes in Mathematics, v. 723, Springer-Verlag, Berlin, 1979.

5. W. Heinzer and J. Ohm, Locally Noetherian commutative rings, Trans. Amer. Math. Soc., 158 (1971), 273-284.

6. E. Matlis, Cotorsion Modules, Mem. Amer. Math. Soc., No. 49, 1964.

$7 . \quad$, Decomposable modules, Trans. Amer. Math. Soc., 125 (1966), 147-179.

8. __ Torsion-free modules, Chicago Lectures in Mathematics, 1972, University of Chicago Press, Chicago.

9. B. Stenström, Rings of Quotients, Die Grundlehren der mathematischen Wissenschaften, v. 217, 1975, Springer-Verlag, Berlin.

10. O. Zariski and P. Samuel, Commutative Algebra, Vol. II, Van Nostrand Co., Princeton, N. J., 1960.

Received July 30, 1981 and in revised form March 15, 1982.

UNIVERSITY OF IDAHO

Moscow, ID 83843 



\section{PACIFIC JOURNAL OF MATHEMATICS}

\section{EDITORS}

Donald BabbitT (Managing Editor)

University of California

Los Angeles, CA 90024

Hugo Rossi

University of Utah

Salt Lake City, UT 84112

C. C. Moore and Arthur Ogus

University of California

Berkeley, CA 94720
J. DugunduI

Department of Mathematics

University of Southern California

Los Angeles, CA 90089-1113

R. FinN and H. SAmelson

Stanford University

Stanford, CA 94305

\section{ASSOCIATE EDITORS}
R. ARENS
E. F. BECKENBACH
B. H. NEUMANN
F. WOLF
K. YosHIDA (1906-1982)

\section{SUPPORTING INSTITUTIONS}

UNIVERSITY OF ARIZONA

UNIVERSITY OF BRITISH COLUMBIA

CALIFORNIA INSTITUTE OF TECHNOLOGY

UNIVERSITY OF CALIFORNIA

MONTANA STATE UNIVERSITY

UNIVERSITY OF NEVADA, RENO

NEW MEXICO STATE UNIVERSITY

OREGON STATE UNIVERSITY
UNIVERSITY OF OREGON

UNIVERSITY OF SOUTHERN CALIFORNIA

STANFORD UNIVERSITY

UNIVERSITY OF HAWAII

UNIVERSITY OF TOKYO

UNIVERSITY OF UTAH

WASHINGTON STATE UNIVERSITY

UNIVERSITY OF WASHINGTON 


\section{Pacific Journal of Mathematics}

Vol. 107, No. $1 \quad$ January, 1983

John Kelly Beem and Phillip E. Parker, Klein-Gordon solvability and the

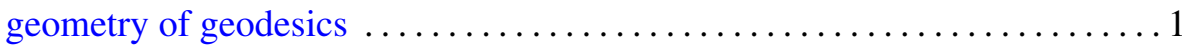

David Borwein and Amnon Jakimovski, Transformations of certain

sequences of random variables by generalized Hausdorff matrices ..... 15

Willy Brandal and Erol Barbut, Localizations of torsion theories . . . . . . . 227

John David Brillhart, Paul Erdős and Richard Patrick Morton, On sums

of Rudin-Shapiro coefficients. II ........................... 39

Martin Lloyd Brown, A note on tamely ramified extensions of rings $\ldots \ldots \ldots 71$

Chang P'ao Ch'ên, A generalization of the Gleason-Kahane-Żelazko

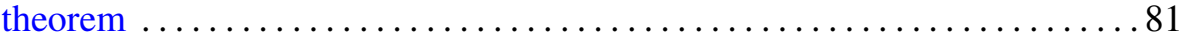

I. P. de Guzman, Annihilator alternative algebras $\ldots \ldots \ldots \ldots \ldots \ldots$. . . . 89

Ralph Jay De Laubenfels, Extensions of $d / d x$ that generate uniformly

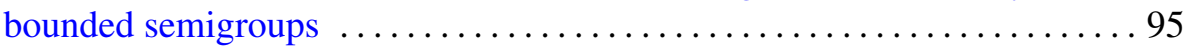

Patrick Ronald Halpin, Some Poincaré series related to identities of $2 \times 2$

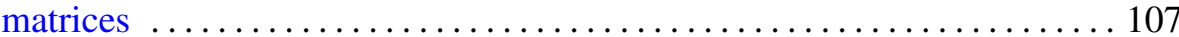

Fumio Hiai, Masanori Ohya and Makoto Tsukada, Sufficiency and

relative entropy in $*$-algebras with applications in quantum systems . . . 117

Dean Robert Hickerson, Splittings of finite groups $\ldots \ldots \ldots \ldots \ldots \ldots \ldots 14$

Jon Lee Johnson, Integral closure and generalized transforms in graded

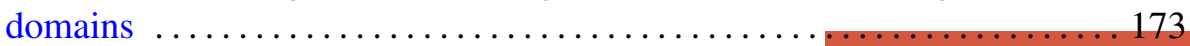

Maria Grazia Marinari, Francesco Odetti and Mario Raimondo, Affine

curves over an algebraically nonclosed field . ................. 179

Douglas Shelby Meadows, Explicit PL self-knottings and the structure of

PL homotopy complex projective spaces $\ldots \ldots \ldots \ldots \ldots \ldots \ldots \ldots \ldots$

Charles Kimbrough Megibben, III, Crawley's problem on the unique

$\omega$-elongation of $p$-groups is undecidable .................... 205

Mary Elizabeth Schaps, Versal determinantal deformations $\ldots \ldots \ldots \ldots 213$

Stephen Scheinberg, Gauthier's localization theorem on meromorphic

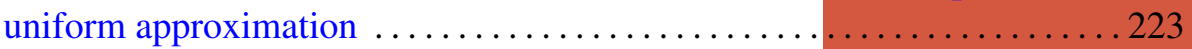

Peter Frederick Stiller, On the uniformization of certain curves . . . . . . . 229

Ernest Lester Stitzinger, Engel's theorem for a class of algebras . . . . . . . . 245

Emery Thomas, On the zeta function for function fields over $F_{p} \ldots \ldots \ldots 251$ 